\title{
RELACIONES FUNCIONALES ENTRE SALUd MENTAL Y CAPITAL SOCIAL EN VÍCTIMAS DEL CONFLICTO ARMADO Y PERSONAS EN SITUACIÓN DE POBREZA*
}

\section{FUNCTIONAL RELATIONSHIPS BETWEEN MENTAL HEALTH AND SOCIAL CAPITAL IN VICTIMS OF ARMED CONFLICT AND PEOPLE IN POVERTY}

\author{
Aura Alicia Cardozo Rusinque** Omar Fernando Cortés-Peña *** Y \\ MaILYN CAStro MONSALVO****
}

\begin{abstract}
*Investigación financiada por la Universidad de la Costa. Barranquilla - Colombia.
**Doctora en Psicología, Magister en Estudios Políticos y Psicóloga. Docente Investigadora en la Facultad de Psicología de la Universidad de la Costa. E-Mail: acardozo@cuc.edu.co

***Doctorando en Psicología, Magister en Psicología, Especialista en Psicología del Consumidor y Psicólogo. Editor de la Revista Cultura Educación y Sociedad y Docente Investigador en la Facultad de Psicología de la Universidad de la Costa. E-Mail: ocortes3@cuc.edu.co

****Estudiante en Práctica Profesional X Semestre en el Semillero de Investigación de la Facultad de Psicología de la Universidad de la Costa. E-Mail: mcastro25@cuc.edu.co

Los autores agradecen a la Facultad de Psicología de la Universidad de la Costa, al Programa de Doctorado en Psicología de la Universidad del Norte y especialmente al Dr. Amalio Blanco de la Universidad Autónoma de Madrid y a la Dra. María

Amarís de la Universidad del Norte sus valiosas orientaciones conceptuales para el desarrollo del estudio.

Facultad de Psicología de la Universidad de la Costa Universidad del Norte

Unidad de Atención y Reparación Integral a Víctimas. Universidad de la Costa, Calle 58 \# 55 - 66. Barranquilla, Colombia.
\end{abstract}

\section{RESUMEN}

Se realizó una investigación que tuvo como objetivo establecer las relaciones funcionales entre salud mental y capital social en personas que han sido víctimas del desplazamiento forzado en el marco del conflicto armado y personas en condición de pobreza. El estudio fue de tipo exposfacto retrospectivo y participaron 380 adultos, clasificados en dos grupos: 190 en situación de desplazamiento y 190 en situación de pobreza. Se administraron los siguientes instrumentos: la Adaptación Española de las Escalas de Bienestar Psicológico de Ryff (Díaz, Rodríguez-Carvajal, Blanco, Moreno-Jiménez, Gallardo, Valle \& Van Dierendonk, 2006), la Escala de Satisfacción con la Vida (Diener, 1994), la Escala de Bienestar
Social (Keyes, 1998) y el Barómetro del Capital Social (Sudarsky, 1999). Posteriormente, se realizó un análisis con el software SPSS 18 para establecer las correlaciones y relaciones funcionales de las variables en los grupos de estudio. Los hallazgos dan cuenta de las correlaciones significativas entre las dimensiones constitutivas del capital social y la salud mental. En segunda instancia se presentan las relaciones funcionales, implementando los modelos de ecuaciones estructurales en cada grupo, asumiendo una relación funcional positiva entre el capital social y la salud mental, que se mantiene en cada grupo estudiado, siendo superior en el grupo de desplazados $(\beta=.56)$ en comparación con el grupo de pobreza $(\beta=.41)$. Estos hallazgos permiten evidenciar que las relaciones funcionales analizadas es- 
tructuralmente entre capital social y salud mental son directamente proporcionales y constituyen un insumo para el desarrollo de programas de intervención psicosocial orientados a la mejoría de la calidad de vida y el empoderamiento social comunitario.

Palabras clave: Capital social; Salud mental; Desplazamiento forzado; Pobreza; Modelos de ecuaciones estructurales.

\section{ABSTRACT}

This paper informs an investigation which had as objective to establish the functional relationships between mental health and social capital in people who have been victims of forced displacement in the context of the armed conflict, and people in poverty conditions. The social capital is understood from the proposal of Putnam (1993), such as the commitment that occurs between actors and social groups where there are networks and standards that facilitate collaboration, the commitment and confidence; through which you can set changes. For the present study, account is taken of the observable variables, measures through the Barometer of Social Capital developed by Sudarsky (2007) and worked in the third measurement of social capital in 2011, in the report presented by Hurtado, García, and Copete (2013), which gives an account of three factors: Solidarity, consolidation of groups and the construction of social fabric (Ksocial), institutional trust and social control (Confie) and credibility in sources of non-validated information (Fenoval). Mental health in research responds to the model of the entire state of health proposed by Keyes (2005b),which assumes the understanding of mental health as a state of well-being, more than the absence of disease, composed of three factors that for terms of the study are the observable variables that make part of the latent variable of mental health, these are: Spanish Adaptation of the Scales of Psychological Well-being of Ryff (Díaz, Rodríguez-Carvajal, Blanco, Moreno-Jiménez, Gallardo, Valle \& Van Dierendonk, 2006) which focuses on the personal development, by which envisaged dimensions like the autoaceptación, positive relationships, autonomy, the domain of the environment, the personal growth and the project of life (Blanco \& Valera, 2007); the Subjective Well-being
(Diener, 1984), constitutes the cognitive and affective evaluations that a person generates around to his life and converges the aspects of the satisfaction and the positive emotions-negative (Blanco \& Valera, 2007) and Social Welfare (Keyes, 1998), understood as the value that we of the circumstances and the operation that we have within the society. Includes items such as integration, acceptance, the contribution, the update and the social coherence. The study was retrospective exposfact type and counted 380 adults, classified in two groups: 190 in situation of displacement and 190 in situation of poverty. The instruments were the Scales Psychological Wellbeing (Ryff, 1989); Satisfaction with Life (Diener, 1994); Social Welfare (Keyes, 1998), and the Barometer of Social Capital (Sudarsky, 1999). Subsequently, an analysis was carried out with the SPSS software 18 to establish correlations and functional relationships of the variables in the study groups. The findings give account of the significant correlations between the constituent dimensions of social capital and mental health. In second instance presents the functional relationships implementing structural equation models in each group, assuming a positive functional relationship between social capital and mental health, which is maintained in each of the groups of study, being higher in the group of displaced persons $(\beta=.56)$ in comparison with the group of poverty $(\beta=.41)$. These findings allowed to demonstrate that the functional relationships analyzed structurally between social capital and mental health are directly proportional and constitute an input to the development of psychosocial intervention programs aimed at the improvement of the quality of life and social empowerment community. This research is a systematic input of structural analysis of the functional relationships between Social Capital and Mental Health, to strengthen the horizon of future studies that should be directed to deepen the analysis of the complex reality that people live in a situation of vulnerability, such as is the case of those who have experienced the forced displacement by effect of the armed conflict and those who have lived in poverty.

Key words: Social capital; Mental health; Forced displacement; Poverty; Functional relationships; Structural equation models. 


\section{INTRODUCCIÓN}

Cada vez hay un mayor reconocimiento de la importancia del contexto social en el bienestar humano, alejándose de la concepción tradicional que ponía el énfasis en el individuo y sus propios procesos, para dar cuenta de la necesidad de reconocer que somos seres históricos y estamos situados en una cultura $\mathrm{y}$ en unas circunstancias (Blanco \& Valera, 2007), lo cual impacta en la vida tanto del sujeto como de su grupo familiar y su comunidad y la sociedad en su totalidad (Cardozo, Amarís \& Blanco, 2015).

Actualmente existen múltiples conflictos armados que azotan a millones de personas en el mundo, dando lugar entre otros flagelos, al desplazamiento forzado, una de las crisis humanitarias de mayor impacto en una sociedad. Según las cifras presentadas por ACNUR (2015), en el mundo se reportan 59.5 millones de víctimas entre las que han padecido el desplazamiento interno (dentro de las propias fronteras), refugiados (o personas que se han visto obligadas a salir de las fronteras de su territorio) y las personas en exilio. Colombia es un país que ha sido atravesado sistemáticamente por la violencia, cargando actualmente el lastre de tener activo el conflicto armado más largo del mundo, con más de 50 años, lo que ha dado lugar a más de seis millones de personas que se encuentran en esta condición, lo que ubica al país en el lugar de la segunda crisis humanitaria más grande del mundo (ACNUR, 2012; OMS, 2013).

La población desplazada entra a su vez a formar parte del contingente de personas en situación de pobreza, dado que el $98 \%$ de dicha población es a su vez pobre, lo cual se corrobora en parte con el informe del Departamento Administrativo Nacional de Estadística (DANE, 2014), que reporta un $30.6 \%$ en situación de pobreza, de los cuales el $9.1 \%$ se encuentra bajo la línea de indigencia, esto es corroborado por la CEPAL (2014) que informa que se presenta una concentración de capital, medida con un Coeficiente Gini $(C G=.539)$. Esto evidencia un grado de desigualdad social importante, vul- nerando así sistemáticamente los derechos humanos y sometiendo a millones de personas a la exclusión social, sin ingresos ni capacidades para satisfacer las necesidades, o como falta de capacidad y libertad (Sen, 2011), aspectos que se vinculan con la injusticia social y la imposibilidad de participar en la vida comunitaria, generando así la exclusión social.

Resulta relevante que en el marco del conflicto armado, la población civil es la que resulta más afectada, por cuanto queda inmersa en medio del fuego cruzado y se constituye en blanco de los grupos armados (Beristain, Dona, Páez, Pérez-Sales \& Fernández, 1999). Esta situación ha afectado a la población más vulnerable en todos los niveles posibles, desde el económico, social y político, hasta en la construcción de las relaciones cotidianas, la capacidad de trabajar conjuntamente, de confiar en el otro (Sudarsky, 2008), establecer vínculos saludables y construir comunidad (Ramos-Vidal, Holgado \& Maya-Jariego, 2014), contar con redes sociales de apoyo y capital social (Palacio, Abello, Madariaga \& Sabatier, 1999) y acceder al bienestar físico, psicológico y social (Abello et al., 2009).

En este marco se han realizado estudios que evidencian que el capital social tiene una relación positiva y altamente significativa con la salud mental y está particularmente asociado al apoyo social y su participación en redes sociales es beneficiosa, lo cual atenúa los acontecimientos dolorosos de la vida (Hurtado, Kawachi \& Sudarsky, 2011; Kawachi, Subramanian \& Kim, 2008; Kripper \& Sapag, 2009; Sudarsky, 2008; Vargas, 2002).

Esta relación es motivada por el creciente interés que ha despertado la concepción que indica que la salud mental no es un fenómeno eminentemente subjetivo e individual, en contraste con la concepción clínica tradicional de la Psicología, dando espacio a una mirada más integral, que atiende la complejidad del ser humano desde todas sus aristas y reconoce las dimensiones psicológicas y sociales. Desde que la OMS (1948) estableció que la salud es más que la ausencia de en- 
fermedad, es la presencia de salud física, psicológica y social, esta perspectiva generó un proceso de fundamentación consecutiva en el marco de las conferencias mundiales sobre la prevención y promoción de la salud con una mirada biopsicosocial, hacia la perspectiva positiva de la salud mental (OMS, 1978, 1986, 1997, 2004, 2005, 2013).

Keyes (2005b) por su parte, formula el Modelo del Estado Completo de Salud (Complete State Model of Health) fundamentado sobre dos axiomas comprobados, que se apoyan en el siguiente supuesto teórico: la salud mental es

"un síndrome de síntomas de hedonía y funcionamiento positivo operacionalizado por medidas de bienestar subjetivo de las percepciones y valoraciones que las personas hacen de su vida y de la calidad de su funcionamiento en la vida" (p. 540).

Esta perspectiva es abordada posteriormente en los trabajos de Paredes, ParedesDíaz, Blanco, Sutil y Schweiger (2007). Esta perspectiva positiva de la salud mental ha sido fundamentada por autores tales como Martín-Baró (1988) para quien la salud mental es

"una dimensión de las relaciones entre las personas y grupos más que un estado individua" (p. 505).

También está fundamentada por Diener (1984) con el concepto de bienestar subjetivo, Ryff (1989) con el concepto de bienestar psicológico, Keyes (1998) con el modelo de bienestar social y posteriormente con el modelo completo de salud (Keyes, 2005a).

Posteriormente se avanzó en la consolidación de una comprensión más integral de los problemas humanos, teniendo en cuenta aspectos psicosociales que inciden en las dinámicas de las relaciones entre las personas, los grupos, las comunidades y de estos con las instituciones en las que están inmersos (Blanco \& Amarís, 2014; Cardozo, Amarís \& Blanco, 2015; Cortés, 2015).

En estos términos es el bienestar uno de los conceptos centrales, teniendo en cuenta las tres dimensiones: psicológica, subjetiva y social y alimentado por dos tradiciones, la hedónica y la eudaimónica.

"La salud mental tiene una relación recíproca con el bienestar y la productividad de una sociedad y sus miembros" (OMS, 2004, p. 23).

Desde esta perspectiva, la salud mental es un recurso individual que contribuye a la calidad de vida, la capacidad de mantener relaciones mutuamente satisfactorias y duraderas, expresadas en la cohesión social que evidencia desarrollo y prosperidad económica de las comunidades.

Esta interacción entre los ámbitos individuales y los propios del entramado social, argumentan la consolidación del Modelo del Capital Social asociado con la perspectiva del Modelo de Salud Mental. A partir de los trabajos de Sudarsky (1999) y Hurtado y colaboradores (2011) se analizan los factores asociados al entramado de las relaciones sociales y su incidencia en la salud mental. En este contexto, surge la aproximación conceptual del presente artículo frente al capital social, el cual pone de manifiesto la importancia del entramado social en el nivel mesosocial, cuyo centro está en la creación de redes de confianza, solidaridad, cooperación y reciprocidad que posibilitan la organización social y la asociatividad cívica (Durston, 2002; Putnam, 1993).

Este estudio se fundamenta en el cambio de paradigma orientado a reconocer el impacto de las relaciones sociales y de las condiciones estructurales en la salud mental, la felicidad y el bienestar del ser humano situado, contextualizado e histórico, que ha sido expuesto a los efectos de la violencia y las condiciones de la pobreza (Blanco \& Valera, 2007; Abello et al., 2009).

En este orden de ideas, resulta de especial interés el abordaje del capital social, concepto que desde la propuesta teórica de Putnam (1993) se asocia con las características de la organización social, entre las que se cuentan aspectos como las redes sociales, las normas y la confianza, mediante las cuales se facilita 
la cooperación para la búsqueda de un beneficio mutuo, sumado a la propuesta de Durston (2000) que lo identifica como una forma comunitaria o colectiva, que va más allá de las relaciones de confianza y reciprocidad entre individuos y sus redes interpersonales, constituyéndose en una mediación para la participación y el empoderamiento y como la capacidad de participación cívica, política y el compromiso cívico, que abona elementos para la reconstrucción del tejido social.

Por lo expuesto surgió el interrogante central que orientó al estudio realizado: ¿Existen relaciones funcionales entre las dimensiones de la salud mental (bienestar subjetivo, psicológico y social) y las dimensiones del capital social (confianza, cooperación, reciprocidad y solidaridad) en personas que viven bajo condiciones de vulnerabilidad por su previa exposición al desplazamiento forzado y por la situación social y económica de pobreza?

Es importante resaltar que las personas desplazadas no solo han estado expuestas a una experiencia traumática que tiende a cambiar las creencias sobre el mundo y sobre sí mismos afectando la salud mental (Abello et al., 2009; Blanco \& Amarís, 2014), también se han visto abocadas a abandonar sus entornos, sus redes sociales de apoyo (Palacio et al., 1999). Adicionalmente se ven enfrentadas al miedo, amenaza, persecución y una vez desplazadas se encuentran en contextos hostiles, donde se ven y sienten como extrañas (Bello, 2001).

El capital social tiene sus raíces a principios del Siglo XX con Lida Hanifan (1916), cuando plantea la importancia de las relaciones entre las personas para alcanzar objetivos comunes, los cuales están basados en la buena voluntad, el compañerismo, la empatía y las relaciones sociales, todas ellas posibilitan la acción colectiva. Este presupuesto brinda fundamento a posteriores búsquedas en la construcción del concepto, transitando por diferentes perspectivas teóricas y epistemológicas, que van desde el individualismo metodológico, con autores como Bourdieu (1986), para quien el capital social es un agregado de recursos asociado a una red de relaciones que posibilita la producción de beneficios y recursos que son apropiados individualmente, seguido por Coleman (1994), quien considera el capital social como un recurso productivo de la estructura social, que posibilita el logro de metas comunes, y Putnam (1993) que plantea una concepción más social, visto como un bien público, basado en la confianza, el compromiso cívico, la cooperación, la reciprocidad, los valores y las actitudes políticas, la prosperidad económica y el bienestar social. Putnam, define al capital social como

“aspectos de la organización social tales como confianza, normas y redes, que pueden mejorar la eficiencia de una sociedad al facilitar la acción coordinada" (1993, p. 167).

Estos elementos se suman a las dimensiones de cooperación y reciprocidad generalizada propiciando beneficios colectivos para asegurar bienes económicos y el funcionamiento de la democracia, constituyéndose en un atributo de las comunidades y no solo un recurso de carácter individual (Forni, Siles \& Barreiro, 2004).

En tal sentido el capital social se constituye en un bien público e inmaterial fundamentado en la relaciones sociales y basado en valores como la solidaridad, comportamientos prosociales y cívicos que reflejan la cohesión social y eficacia colectiva. Al respecto Hurtado, García y Copete hacen referencia a las

"características de los grupos sociales, como el grado de confianza interpersonal, normas de reciprocidad y la asociatividad -la membresía a organizaciones voluntarias- que facilitan la acción colectiva" (2013, p. 34).

En concordancia con lo planteado previamente por Díaz-Albertini (2001) el capital social promueve la acción colectiva, así como el autocontrol y la responsabilidad social, en cuanto se constituye en una fuente de acercamiento entre los seres humanos a partir de aspectos como la confianza, la reciprocidad y el respeto a las reglas. Esta argumentación permite construir relaciones de confianza y reciprocidad, que contribuyen a la cohesión, el desarrollo y el bienestar de la sociedad, 
además de favorecer la capacidad de sus miembros para actuar y satisfacer sus necesidades coordinadamente en busca del beneficio mutuo (Alberdi \& Pérez, 2006).

En consecuencia, el capital social comprende el desarrollo de acciones colectivas que favorecen el beneficio mutuo y permite alcanzar objetivos colectivos, fortaleciendo la confianza y la cooperación, promueve la interacción social a través de establecer relaciones de vecindad, amistad y construir comunidad (Arriagada, 2004; González, 2009; Forni et al., 2004; Palacio, Correa, Díaz \& Jiménez, 2003).

En un contexto de violencia y de pobreza extrema es evidente que tanto la salud mental como el capital social serán variables fuertemente afectadas por la vulnerabilidad generada por estas condiciones, impactando la capacidad de las personas, grupos y comunidades de confiar en los otros y en las instituciones (Hurtado et al., 2013).

\section{MÉTOdo}

Para el estudio se realizó un diseño ex post facto retrospectivo con dos grupos, a fin de establecer las relaciones funcionales entre capital social y salud mental desde la perspectiva positiva (Cardozo et al., 2015). Los diseños de corte ex post facto retrospectivo con grupo cuasi control, según León y Montero (2004), comprenden estudios en los que las limitaciones para el contraste de las relaciones causales vienen dadas por la imposibilidad de manipular la variable independiente.

DEFINICIÓN DE LAS VARIABLES CENTRALES DEL ESTUDIO

Desde la propuesta de Putnam (1993) el capital social se entiende como el compromiso que se presenta entre actores y grupos sociales en donde existen redes y normas que facilitan la colaboración, el compromiso y la confianza mediante los cuales se pueden establecer cambios. En palabras de Putnam (1995, p. 67): "las características de la organización social, tales como las redes, las normas y la confianza, que facilitan la coordinación y la cooperación para el beneficio mutuo".

Para Urteaga (2013) podría entenderse como un componente que facilita la acción colectiva y permite reconciliar el interés individual con el social, mediante el asociacionismo, el compromiso cívico y la cohesión social.

Se tuvieron en cuenta las variables observables, evaluadas con el Barómetro del Capital Social desarrollado por Sudarsky (2007), utilizado en la tercera medición del capital social en el 2011, y presentado en el informe de Hurtado y colaboradores (2013), el cual da cuenta de tres factores: el factor asociado a la medida del tejido social (ksocial) que contiene nociones teóricas del capital social, tales como el grado de solidaridad generalizada presente en la sociedad, el estado de las relaciones entre iguales, la consolidación de círculos sociales y la vinculación centro periferia de una sociedad y se evalúa igualmente la relación de la sociedad con la institucionalidad. El factor confie comprende la confianza en instituciones del Estado. Finalmente, el factor fenoval evalúa las creencias en fuentes de información como son los medios de comunicación, la prensa y representantes del Estado. Este constructo considera implícitamente elementos como las creencias de los ciudadanos asociadas a la calidad y suficiencia de la información ofrecida por diversas fuentes, la responsabilidad sobre lo público y el impacto de los medios de comunicación en la formación y acumulación de capital social (Hurtado et al., 2013).

Se presentan a continuación los tres modelos, todos de primer grado y con la validez estadística requerida. En la Tabla 1 se identifican los niveles estadísticos de bondad de ajuste y los criterios de referencia son el $C h i$ cuadrado $\left(\chi^{2} \geq .05\right)$, el Índice de Bondad de ajuste comparativo $(C F I \geq .95)$, la raíz del residuo cuadrático promedio $S$ - $R M R$ que debe ser próximo a cero y la raíz de residuo cuadrático promedio de aproximación $(R M S E A \leq .08)$, asumidos como indicadores 
de calidad del ajuste de los modelos estructurales en el presente estudio. La Tabla 1 informa valores estimados en los modelos de medición estructural del capital social y la salud mental.

La salud mental abordada en la investigación responde al Modelo del Estado Completo de Salud propuesto por Keyes (2005b), que asume el entendimiento de la salud mental como un estado de bienestar, más que la ausencia de enfermedad, compuesto por tres factores que constituyen las variables observables que forman parte de la variable latente de salud mental y ellos son: Bienestar psicológico (Keyes, Shmotkin \& Ryff, 2002), centrado en el desarrollo personal, por lo que contempla dimensiones como la autoaceptación, las relaciones positivas, la autonomía, el dominio del entorno, el crecimiento personal y el proyecto de vida (Blanco \& Valera, 2007). El Bienestar subjetivo (Diener, 2005) constituye las evaluaciones cognitivas y afectivas que una persona genera en torno a su vida y convergen los aspectos de la satisfacción y los afectos positivo - negativo (Blanco \& Valera, 2007) y el bienestar social (Keyes, 1998), entendido como la valoración que hacemos de las circunstancias y el funcionamiento que tenemos dentro de la sociedad. Contempla elementos como la integración, la aceptación, la contribución, la actualización y la coherencia social.

\section{PARTICIPANTES}

La muestra se configuró con 380 participantes mayores de 18 años, de nacionalidad colombiana. La selección de los sujetos se realizó a través de un muestreo de tipo no probabilístico por criterio de tipo experto, dada la naturaleza del estudio.

La muestra se dividió en dos grupos. El Grupo Desplazados estuvo integrado por 190 participantes que vivieron la experiencia del desplazamiento forzado en el marco del conflicto armado y actualmente se encuentran vinculados a la Unidad de Atención y Reparación Integral a Víctimas, Departamento del Atlántico al norte de Colombia y el Grupo
Pobreza, conformado por 190 participantes en condición de pobreza, ubicados en barrios subnormales o en estrato uno del área metropolitana de Barranquilla que tenían vinculación con instituciones de apoyo al desarrollo social.

Todos los participantes ofrecieron su consentimiento para participar en la investigación.

\section{INSTRUMENTOS}

Para la medición de cada variable se utilizaron las escalas de Salud Mental (Modelo del Estado Completo de Salud - Keyes, 2005b), constituidas por tres dimensiones:

La Adaptación Española de las Escalas de Bienestar Psicológico de Ryff (Díaz, Rodríguez-Carvajal, Blanco, Moreno-Jiménez, Gallardo, Valle \& Van Dierendonk, 2006) consta de 29 ítems y tiene una fiabilidad igual a .80. La escala evalúa aspectos como autoaceptación, relaciones positivas con los otros, autonomía, dominio del entorno, objetivos vitales y crecimiento personal.

La Escala de Satisfacción con la Vida (Diener, 2005) consta de cinco ítems y tiene una fiabilidad igual a .86. Los ítems son: (1) El tipo de vida que llevo se parece al tipo de vida que siempre soñé llevar. (2) Las condiciones de mi vida son excelentes. (3) Estoy satisfecho con mi vida. (4) Hasta ahora he obtenido las cosas importantes que quiero en la vida y (5) Si pudiera vivir mi vida de nuevo, me gustaría que todo volviese a ser igual.

La adaptación al español (Díaz et al., 2006) de la Escala de Bienestar Social (Keyes, 1998) que consta de 25 ítems y tiene una fiabilidad igual a .83. La escala evalúa dimensiones tales como: (1) Integración social, (2) Adaptación social, (3) Contribución social, (4) Actualización social y (5) Coherencia social.

Para medir el Capital Social se utilizó el Barómetro del Capital Social (Sudarsky, 2007), el cual fue ajustado para la medición 
nacional de capital social realizada en el año 2011. El cuestionario evalúa tres factores: el tejido social, confianza en instituciones y confianza en la información. Consta de 325 ítems distribuidos en 10 dimensiones. La confiabilidad del Barómetro de Capital Social fue estimada con el coeficiente Alpha de Cronbach en .849 .

\section{Procedimiento}

Atendiendo los objetivos planteados para este estudio se han dispuesto siete fases: se realizó una revisión bibliográfica tomando como aspectos centrales la teoría de capital social y la salud mental desde la perspectiva positiva, se describieron los supuestos en los que se basan las hipótesis de investigación y se planteó el modelo teórico que mostrara las relaciones funcionales que se presumen existen entre el capital social y la salud mental.

Se comprobó la confiabilidad y validez de las herramientas a utilizar mediante el análisis factorial realizada con el SPSS versión 18. Se determinó el proceso para realizar la recolección de los datos mediante la aplicación de cuadernillos con las escalas enunciadas, con una aplicación heterónoma y con el apoyo de personal capacitado para su aplicación. Se procesaron los datos obtenidos con el paquete de análisis estadístico SPSS versión 18 y se realizó un análisis factorial con la muestra completa, estableciendo el índice de confiabilidad y validez. El análisis de datos presenta una primera fase de corte correlacional entre el capital social y la salud mental y posteriormente se establecieron las relaciones funcionales entre el capital social y la salud mental con el uso de los modelos de ecuaciones estructurales, al interior de cada grupo de estudio.

\section{Resultados}

Se presentan los principales hallazgos derivados del estudio y posteriormente se presentan las relaciones funcionales encontradas en estas mismas variables en los dos grupos, para finalmente establecer las diferencias y semejanzas entre los grupos tanto a nivel de las correlaciones como de las relaciones funcionales.

Como parte del proceso se estimaron los coeficientes de correlación de Pearson entre las variables de estudio y sus dimensiones. En tal sentido las dos variables centrales, capital social y salud mental presentaron altos niveles de consistencia interna con asociaciones directamente proporcionales y estadísticamente significativas al 99\% $(\rho . v .=.00)$ establecidas al interior de cada grupo de estudio.

Para el capital social se obtuvieron los siguientes índices de correlación, para ambos grupos asociados con la consistencia interna frente a sus factores de tejido social: ksocial: .677 para desplazados y .687 para el grupo de pobreza; para confianza en instituciones confie: .821 para desplazados y .843 para pobreza, y para el factor relativo a fe en información no validada fenoval: .785 desplazados y .791 para el grupo de pobreza.

Para salud mental por su parte, los índices de correlación presentaron la siguiente consistencia en sus diferentes dimensiones: .682 para desplazados y .749 para pobreza; bienestar subjetivo: .789 para desplazados y .731 para pobreza, y bienestar social: .686 para desplazados y .719 para pobreza. Lo anterior evidencia una correspondencia entre las dimensiones que constituyen las variables observables de las respectivas variables latentes analizadas en el estudio.

El análisis preliminar de correlaciones muestra que los parámetros estructurales de las escalas de capital social frente a la variable de salud mental, marca una correlación significativa al nivel de $p . v .=.01$, unilateral, tanto para el grupo de personas en situación de pobreza $(r=.227)$, como para el grupo de desplazados $(r=.217)$, lo que traduce que a mayores niveles de capital social, mayores son los niveles de salud mental.

A continuación se profundiza en el análisis de las relaciones funcionales establecidas entre el capital social y la salud mental, teniendo en cuenta de manera central los resultados presentados con mayores niveles de significación, para cada grupo de estudio, con 
los resultados de las correlaciones entre las dimensiones de las variables estudiadas, evidenciando las semejanzas de los datos obtenidos en los puntajes de ambos grupos de estudio.

Las correlaciones para el grupo de personas desplazadas marcaron puntajes estadísticamente débiles, los cuales siguen evidenciando una tendencia marcada en el comportamiento de estas variables, donde las relaciones más fuertes entre las dimensiones de las variables se presentaron entre ksocial y salud mental $(r=.305)$ y confie y bienestar psicológico $(r=.291)$ tal como se presenta detalladamente en la Tabla 2.

Las correlaciones a nivel de las dimensiones de las variables latentes estudiadas para el grupo de pobreza, tal como se evidencia en la Tabla 2, donde se presentan puntajes que aunque son estadísticamente débiles, muestran una tendencia marcada del comportamiento entre estas variables. En ese sentido, la puntuación más fuerte y significativa al nivel (p.v. $=.01$ - unilateral) se presentó entre la dimensión confíe del capital social y la salud mental $(r=.236)$, respectivamente (ver Tabla 3).

La relación funcional entre las variables capital social y salud mental tuvo un peso $(\beta=.56)$ en el grupo que ha experimentado la experiencia del desplazamiento forzado. Se evidencia igualmente que el comportamiento de cada variable latente fue diferente según el grupo. Para el grupo de desplazados la dimensión con mayor peso fue la confianza institucional (confie) $(\beta=.70)$, para el capital social, seguido del tejido social ( $\mathrm{kso}$ cial), siendo de menor peso el factor de fe en medios de información no validada (fenoval).

Con relación a salud mental, el comportamiento de las dimensiones del bienestar, se encuentra que la variable observable de mayor peso es bienestar psicológico $(\beta=.73)$, seguida del bienestar social $(\beta=.54)$ y con menor peso, el bienestar subjetivo $(\beta=.33)$, tal como se puede observar en la Figura 1.

Por su parte, la relación funcional obtenida para las variables capital social y salud mental para el grupo en situación de pobreza, tal como se ilustra en la Figura 2, da los siguientes resultados: el peso de la relación funcional fue $\beta=.41$. El comportamiento de cada dimensión de capital social para este caso, fue de mayor peso: el factor relativo a la confianza institucional (confie) $(\beta=.82)$, seguido del tejido social (ksocial) con un peso $\beta=.63$ y con un mínimo peso de fe en información no validada (fenoval), con un peso $\beta=.16$. Con relación al comportamiento de las variables observables de la salud mental se encuentra que el bienestar psicológico tiene el mayor peso $(\beta=.73)$, que al igual que bienestar social alcanza un peso significativo de $\beta=.72$, mientras que bienestar subjetivo no alcanza un peso significativo ya que solo es de $\beta=.16$.

En términos generales, se evidencia en las relaciones funcionales entre las variables de ambos grupos, que el mayor peso en la relación se encuentra en el grupo que vivió la experiencia del desplazamiento, siendo significativamente mayor que en el grupo en situación de pobreza (Cardozo et al., 2015).

\section{Discusión}

Se presenta un análisis de los resultados de la investigación, con sus alcances, limitaciones y futuras líneas de desarrollo a la luz de los componentes teóricos y contextuales que lo fundamentan y permiten establecer las relaciones que se dan entre el capital social y la salud mental, en personas en situación de pobreza y en víctimas del desplazamiento forzado por el conflicto armado, otorgando especial importancia al componente social de la salud mental.

López y Sánchez (2001) señalan la necesidad de incluir los fundamentos sociales y estructurales como la posición y la integración social, donde resultan de vital importancia aspectos como el apoyo social, que abarcan aspectos más amplios que los que se habían asociado a la salud mental desde los modelos teóricos tradicionales, elementos que desde la OMS (2004) han sido reiteradamente formulados y enriquecidos con los aportes de Diener (2005), Ryff (1989), Keyes (2005b) y Díaz, Blanco, Horcajo y Valle (2007). 
Los resultados evidencian que existe una relación entre salud mental, abordada desde una perspectiva positiva, a partir del modelo del estado completo de salud de Keyes (2005), con las dimensiones del bienestar psicológico, bienestar subjetivo y bienestar social, con capital social, a partir de los factores propuestos por Sudarsky (2008) definidos como tejido social, confianza en instituciones y fe en información no validada, que se articulan con el capital social cognitivo (confianza, reciprocidad, cooperación y solidaridad) y el capital social estructural (dimensiones institucional, control social, participación cívica y participación política). Estas aproximaciones basadas en análisis factoriales son convergentes con la propuesta de Resett, Costa, Murata y Falcone (2015).

Cabe resaltar que las limitaciones del estudio realizado se derivan de las restricciones para acceder a la muestra de participantes. Sin embargo, los resultados evidenciaron en primera instancia que la relación entre capital social y salud mental, tiende a ser similar en los dos grupos. Adicionalmente no se presentan diferencias estadísticamente significativas, lo cual se atribuye en primera instancia a que ambos grupos están estrechamente ligados a problemas propios de la vulnerabilidad socioeconómica, con independencia del evento traumático sufrido por parte de la población desplazada.

En tal sentido, comparten los problemas propios de la pobreza como la marginación, la falta de oportunidades, la violación sistemática de los derechos humanos, la violencia urbana y particularmente la carencia de recursos y oportunidades, si se tiene en cuenta que un $98 \%$ de la población desplazada se encuentra además en situación de pobreza. Adicionalmente en el $48 \%$ de las familias desplazadas, las madres asumen la jefatura del hogar y un $36 \%$ es menor de 18 años. Por lo anterior, estas familias conforman el 75\% de la población desplazada, consideradas además como especialmente vulnerables (ACNUR, 2015), tanto por su edad como por ser familias mono-parentales.

En segundo lugar los grupos presentan correlaciones débiles entre las dos variables, lo cual se asocia a la relevancia que las personas suelen darle a los factores psicológico y subjetivo, en detrimento del componente social, en razón de la posibilidad de dar respuestas desde su propia capacidad, y encontrando particularmente difícil controlar el aspecto social, como lo evidenciaron Abello y colaboradores (2009). Estos autores encontraron que la población desplazada presenta mayores niveles de restablecimiento psicológico y subjetivo, mientras que hallaron mayor dificultad en el componente del bienestar social, en razón de que no depende solo de sí mismos, sino que está enmarcado en las relaciones sociales, es decir, en el encuentro con el otro y con las instituciones (Cardozo et al., 2015).

Empíricamente quedó en evidencia la relación entre capital social y salud mental, tal como lo afirma Vargas (2002), quien demostró que el capital social y la salud mental tienen relación en diferentes dimensiones y formas, en tanto las relaciones sociales son una fuente importante de bienestar psicológico y social. Asimismo Kripper y Sapag (2009) encontraron que la relación entre capital social y salud mental presenta variaciones, dependiendo de los subtipos o categorías de capital social y las condiciones socioeconómicas y culturales específicas.

Aunque los resultados fueron similares para ambos grupos, se evidencian algunas diferencias ya sea de tendencia o de magnitud, que permiten realizar un análisis acerca del comportamiento particular de las variables observables, es decir, cada dimensión que compone el capital social y la salud mental, para cada uno de los grupos, lo cual se presenta en el siguiente apartado, para posteriormente realizar una comparación que dé cuenta de cómo se manifiestan estas diferencias (Cardozo et al., 2015).

Adicionalmente, se identificó la presencia de dos correlaciones estadísticamente significativas en el grupo de la población desplazada, como son el capital social con el bienestar psicológico y el capital social cognitivo con la salud mental. Este resultado concuerda con los hallazgos de Hurtado y colaboradores (2011) quienes encontraron que el capital social cognitivo está asociado con la salud men- 
tal, en detrimento de la asociación entre capital social estructural con la salud mental.

Frente a la relación funcional entre bienestar psicológico con capital social cognitivo (solidaridad, confianza y reciprocidad), se han presentado evidencias empíricas que estos dos aspectos son los que presentan mayor asociación, tal como lo informan Hurtado y colaboradores (2011), quienes plantearon diferencias entre los efectos de la salud mental del capital social cognitivo y estructural. Igualmente en este contexto se presentó una correlación entre bienestar psicológico y capital social estructural, lo cual concuerda igualmente con los autores mencionados, quienes indican que el capital social estructural posibilita las conexiones y la pertenencia a organizaciones, reduciendo los impactos negativos de acontecimientos vividos.

Se encontró una relación funcional positiva entre capital social cognitivo y bienestar social debido a que las relaciones horizontales o verticales, la solidaridad, la reciprocidad, las actividades sociales del vecindario, la pertenencia a organizaciones de interés, entre otras, generan mayor bienestar a nivel social ya que estos elementos fortalecen los lazos entre quienes conforman el colectivo (Cardozo et al., 2015).

Por otra parte, en el grupo de personas en situación de pobreza, se encontró una asociación entre el capital social estructural (participación en actividades voluntarias, participación cívica y política) y el bienestar psicológico. Afirman que los vínculos sociales, como el apoyo social y la participación en redes sociales, la influencia social y los estados afectivos positivos juegan un papel beneficioso sobre el mantenimiento del bienestar psicológico; aportan además dos modelos (estrés-buffering y principal afecto) en los cuales se acepta que los vínculos sociales juegan un papel beneficioso sobre el mantenimiento del bienestar psicológico.

El capital social es visto como una dimensión integral que articula aspectos como la pertenencia a redes sociales ya que estas se constituyen en uno de los mecanismos de las poblaciones pobres para superar sus problemas (Durston, 2000; Madariaga \& Sierra,
2000). Tal como se evidenció en los resultados, el capital social incide positivamente en la salud mental, en especial frente a la confianza en las instituciones, el desarrollo del potencial humano, la capacidad de ejercer control en el entorno y el verter esperanza en el futuro de la sociedad.

Algunos estudios sugieren similarmente que la capacidad de trabajar en conjunto en pro de la búsqueda de soluciones a problemas comunes permite un mejor desarrollo de las relaciones, una mayor cohesión (Verduin, Smid, Wind \& Scholte, 2014), el decrecimiento de trastornos mentales (Fernández, Manrique, Bojorquez \& Salinas, 2014; Wu, Lu \& Kang, 2014), el aumento de la confianza en el contexto social, la eficiencia colectiva frente a desastres (Wind \& Komproe, 2012), la mayor presencia de emprendimiento y acción social en la comunidad (Sapag \& Kawachi, 2007), las mejorías significativas en la autopercepción favorable del estado de salud, el apoyo social subjetivo, el acercamiento a la familia y la comunidad y el apoyo social (Bilbao, Páez, Da Costa \& Martínez-Zelaya, 2013). Esto ha sido corroborado anteriormente por Rose (2000) bajo el argumento de que las redes de apoyo social implícitas en el capital social proveen apoyo emocional, lo cual reduce la probabilidad de depresión emocional.

\section{Conclusiones}

La asociación entre la salud mental y el capital social es significativa aunque su magnitud es débil, siendo consistente con investigaciones de Kawachi y colaboradores (2008). Sin embargo, se resalta la importancia del componente social en torno a la salud mental, tal como lo propone la OMS (2015), cuando plantea el modelo de análisis integral del ser humano teniendo en cuenta aspectos sociales, económicos, políticos y culturales.

Al respecto, Blanco y Valera (2007) establecen su análisis del ser situado en un contexto histórico y social en concordancia con el reconocimiento de que la sociedad impacta en la psicología humana, acorde con los prin- 
cipios de Durkheim (2012) quien postula que la estructura de la sociedad tiene un fuerte efecto sobre la salud psicológica, así como lo evidenció Martín-Baró (1988) en su análisis de la influencia de la estructura social sobre el comportamiento de las personas y relaciones sociales.

Desde la perspectiva de Landini (2012) se evidencia la importancia del abordaje psicológico en los contextos de intervención social y comunitaria orientados al fomento del asociativismo, el trabajo grupal y la comunicación interpersonal, los cuales también forman parte de los dispositivos que nutren el empoderamiento de las comunidades frente a sus procesos de transformación colectiva en el marco del capital social.

El capital social se constituye en un elemento importante para el restablecimiento de la salud mental, así como la capacidad para enfrentar las situaciones de la vida, a nivel social, contextual, histórico y cultural (OMS, 2004). Esta reflexión se enriquece con la perspectiva de Muñoz, Poblete y Jiménez (2012) en su estudio sobre calidad de vida familiar y bienestar subjetivo, al resaltar el papel fundamental de las interacciones familiares y el desarrollo de redes de apoyo social para fortalecer el bienestar emocional.

Algunas tradiciones de relación sociedadestado tienden al clientelismo y la corrupción, lo cual afecta su compromiso efectivo con el desarrollo social y genera una profunda distorsión del papel que se puede jugar en la sociedad (Putnam, 1993; Sudarsky, 2008). Esta distorsión afecta la percepción del bienestar social (Keyes, 1998, 2005a, 2005b) y es congruente con los aportes derivados de Hurtado y colaboradores (2011). Estas reflexiones se integran a su vez con los aportes de Alonso y Brussino (2012) sobre la valoración de los aspectos relacionados con la justicia procedimental y el análisis del rol que asumen las autoridades legales.

En este orden de ideas, el componente social podría interpretarse como una dimensión que amerita procesos de intervención psicosocial orientados a la construcción del tejido social en interacción con la promoción de la salud mental y la calidad de vida (Hurtado et al., 2011). Complementariamente, Abello y colaboradores (2009) identifican que el bienestar social es el que tiene mayor dificultad para restablecerse, dado que para ello requiere el entramado de relaciones sociales de sus interacciones con un entorno.

En el caso del conflicto armado, la violencia es una expresión consecuente con el horror de la guerra, lo que obstaculiza la capacidad para asumir los retos de la vida en detrimento del componente social, político, económico y cultural. Alonso y Brussino (2012) en su estudio convergen con la percepción sobre el marco de la legitimidad y el ejercicio de la justicia bajo el contexto de la vulnerabilidad social, afectando la eficacia institucional, lo cual se articula con el análisis de las barreras contemporáneas frente al desarrollo del capital social.

Al respecto, Woolcock y Narayan (2000) analizan el capital social como uno de los elementos estudiados en relación con la salud pública que permite la acción colectiva mediante el establecimiento de redes y fortalecimiento de la circulación de información como recursos psicosociales para garantizar el bienestar y la salud mental. Este argumento es consistente con la articulación funcional desarrollada por Cortés (2015) en su estudio sobre bienestar laboral y salud mental y los estudios de Muñoz y colaboradores (2012) sobre calidad de vida familiar y bienestar subjetivo en jóvenes.

Los hallazgos evidencian la importancia que tienen las instituciones estatales en la construcción de capital social, la academia y los sectores privados en el marco de la corresponsabilidad frente al desarrollo social y humano, el fortalecimiento de la identidad nacional, la tolerancia, la construcción de la paz sobre la base de un mundo justo, equitativo y con calidad de vida articulada funcionalmente al desarrollo sostenible (Barreiro, Etchezahar \& Prado-Gascó, 2014; Cortés, 2015; Landini, 2012; Monsegur, Espinosa \& Beramendi, 2014).

El estudio realizado constituye un insumo sistemático derivado del análisis estructural de las relaciones funcionales entre el capital social y la salud mental, en el horizonte de 
estudios futuros que se orienten al análisis de la compleja realidad que afrontan las personas en situación de vulnerabilidad, tal como es el caso de quienes han experimentado el desplazamiento forzado por efecto del conflicto armado y quienes han vivido en situación de pobreza, ahora proyectados a la construcción de una cultura de la paz.
Finalmente, el análisis funcional entre el capital social y la salud mental aporta una nueva perspectiva para el concepto del desarrollo social y la calidad de vida, de la mano con los procesos de crecimiento, empoderamiento y transformación comunitaria, en una nueva cultura de la paz, en el horizonte del desarrollo humano y sostenible.

TABLA 1

INDICADORES DE AJUSTE ASOCIADOS A LOS MODELOS DE MEDICIÓN ESTRUCTURAL DEL CAPITAL SOCIAL Y LA SALUD MENTAL

\begin{tabular}{|c|c|c|c|c|c|}
\hline Modelos de Medición Estructural & $\chi^{2}$ & $p-\chi^{2}$ & $\mathrm{CFI}$ & RMSEA & S-RMR \\
\hline $\begin{array}{l}\text { Modelo de Medición de Capital Social } \\
\text { Modelo de Medición de Salud Mental }\end{array}$ & $\begin{array}{l}.306 \\
.105\end{array}$ & $\begin{array}{l}.580 \\
.745\end{array}$ & $\begin{array}{l}1 \\
1\end{array}$ & $\begin{array}{l}0 \\
0\end{array}$ & $\begin{array}{l}.0079 \\
.0057\end{array}$ \\
\hline
\end{tabular}


Cardozo Rusinque, Cortés-Peña y Castro Monsalvo

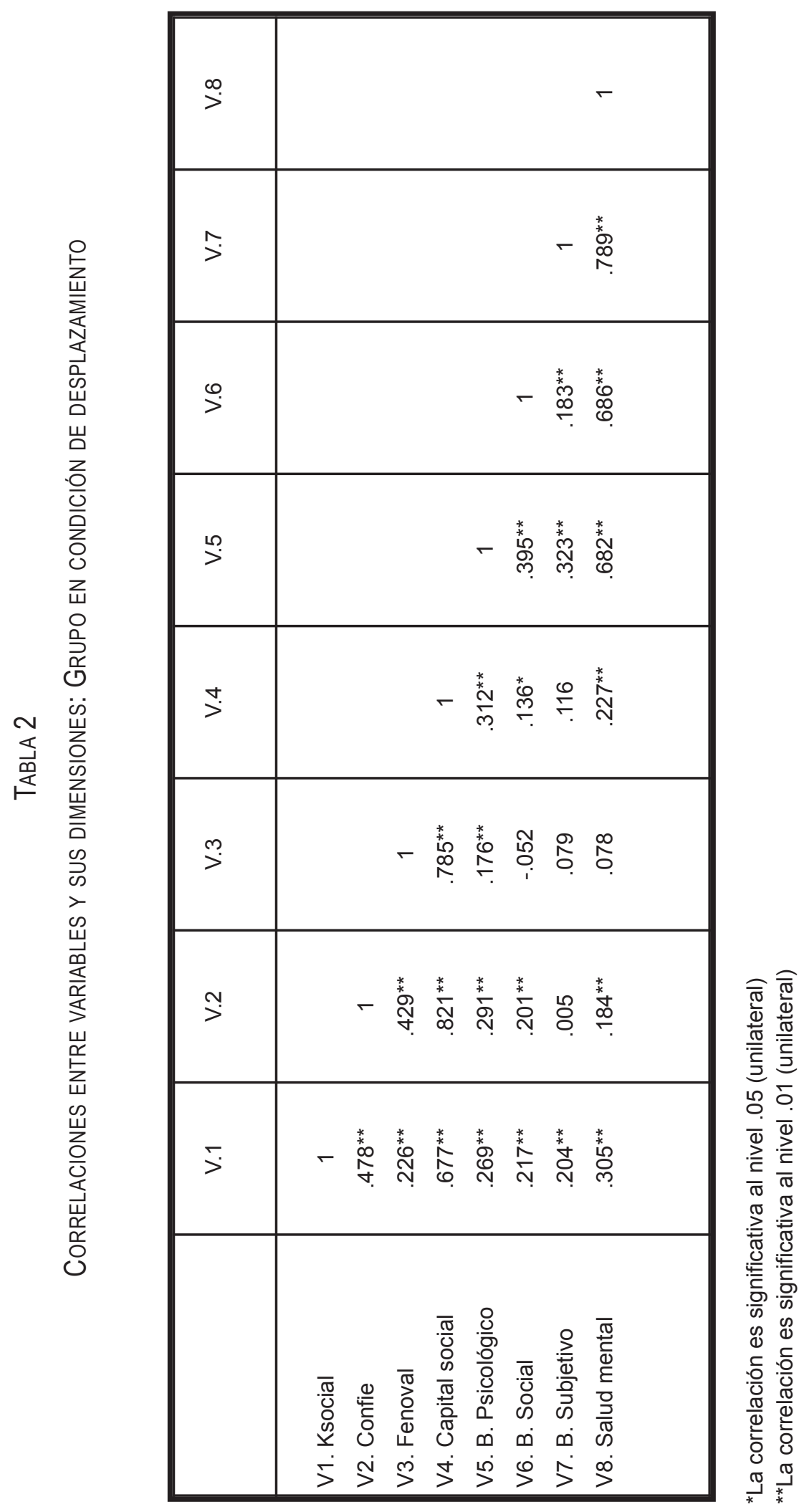




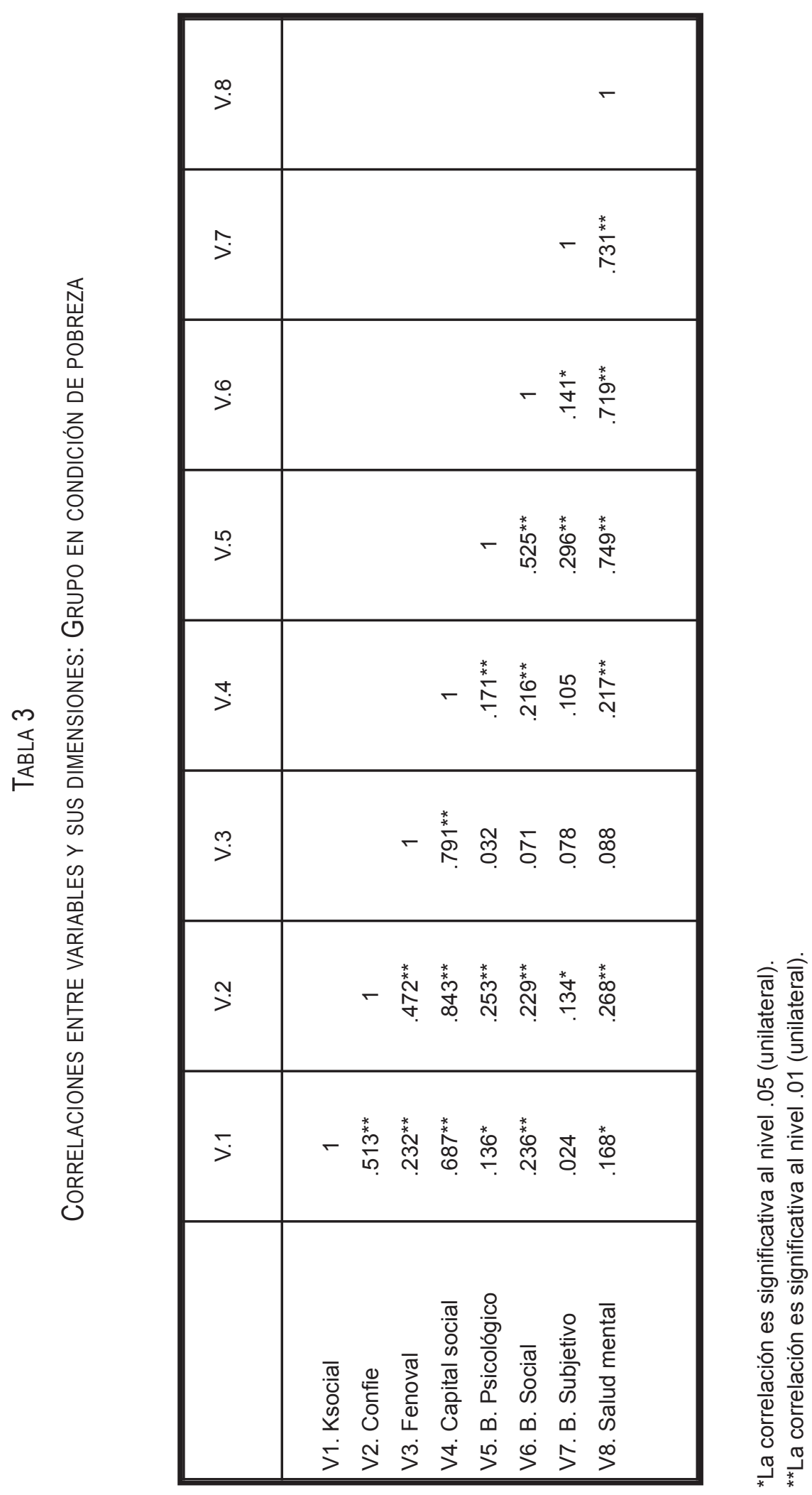


FIGURA 1

Modelo estructural CAPITAL SOCIAL - SALUd MENTAL: GRUPO EN CONDICIÓN DE DESPLAZAMIENTO

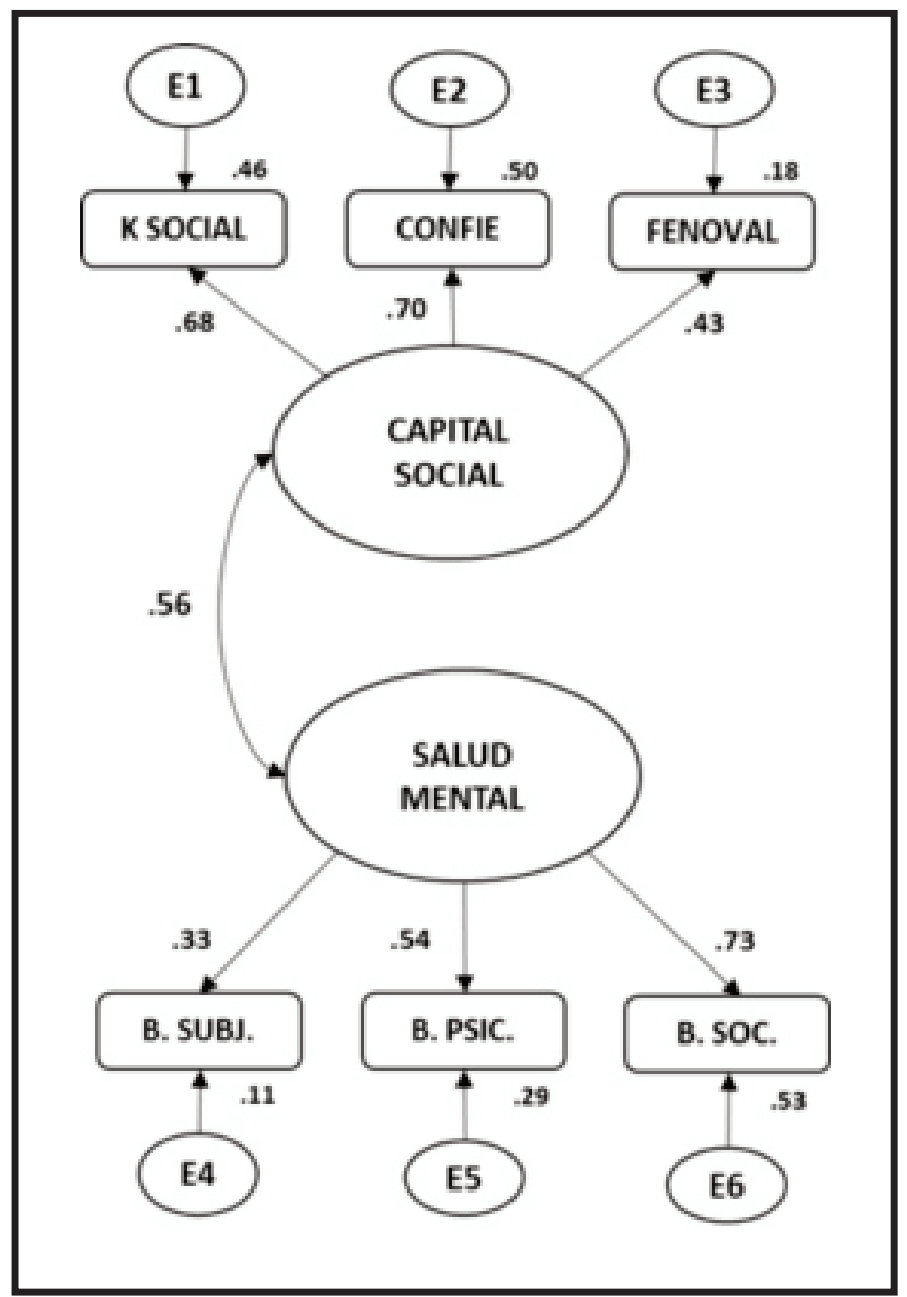


FIGURA 2

ModelO ESTRUCTURAL CAPITAL SOCIAL - SALUD MENTAL: GRUPO EN CONDICIÓN DE POBREZA

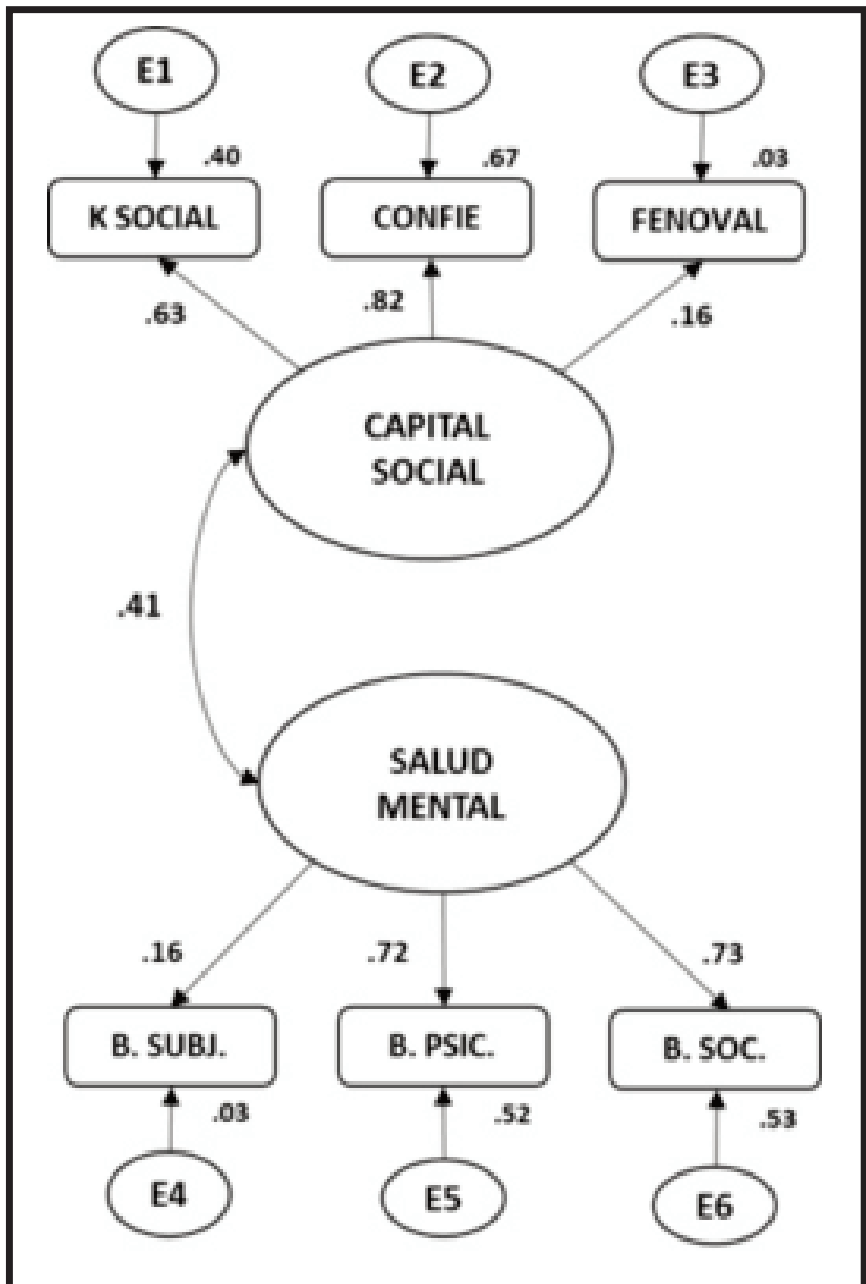




\section{REFERENCIAS BIBLIOGRÁFICAS}

Abello, R., Amarís, M., Blanco, A., Madariaga, C., Manrique, K., Martínez, M., Turizo, Y. \& Díaz, D. (2009). Bienestar y trauma en personas adultas desplazadas por la violencia política [Wellbeing and trauma in adults displaced by political violence]. Universitas Psychologica, 8(2), 455-470.

Alberdi, J. \& Pérez, K. (2006). Capital social. Diccionario de acción humanitaria y cooperación al desarrollo [Social capital. Dictionary of humanitarian action and development cooperation]. Barcelona: Universidad del País Vasco. Recuperado el 11 de octubre de 2015 de http:// www.dicc.hegoa.ehu.es/listar/mostrar/29.

Alonso, D. \& Brussino, S. (2012). Legitimidad y percepciones generales de justicia de las autoridades legales de Córdoba (Argentina): Un abordaje desde las valoraciones de justicia procedimental [Legitimacy and general perceptions of judicial authorities from Córdoba (Argentina): An approach from procedural justice perceptions]. Interdisciplinaria, 29(2), 287-304. Recuperado el 5 de mayo de 2016 de http:// www.redalyc.org/articulo.oa?id=18026361007

Alto Comisionado de las Naciones Unidas para los Refugiados [ACNUR] (2012). Un año de crisis [A year of crisis]. Documento de trabajo. Recuperado el 5 de mayo de 2015 de http://acnur.es/ PDF/tendenciasglobales_2011_spa_201206191 51451.pdf.

Alto Comisionado de las Naciones Unidas para los Refugiados [ACNUR] (2015). Mundo en guerra [World in war]. Documento de trabajo. Recuperado el 5 de mayo de 2015 de http://www. acnur.org/t3/fileadmin/Documentos/Publicacio nes/2015/10072.pdf?view=1.

Arriagada, I. (2004). Estructuras familiares, trabajo y bienestar en América Latina [Family structures, work and well-being in Latin America]. Naciones Unidas-CEPAL, División de Desa- rrollo Social. Recuperado el 19 de abril de 2014 de http://www.cepal.org/dds/noticias/paginas/9 /19679/IArriagada.pdf.

Barreiro, A., Etchezahar, E. \& Prado-Gascó, V. (2014). Creencia global en un mundo justo: Validación de la Escala de Lipkus en estudiantes universitarios de la ciudad de Buenos Aires [Global belief in a just world: Validation of the Lipkus Scale in university students from city of Buenos Aires]. Interdisciplinaria, 31(1), 57-71. Recuperado Recuperado el 7 de mayo de 2016 de http://www.scielo.org.ar/pdf/interd/v31n1/ v31n1a04.pdf.; https://doi.org/ 10.16888/interd. 2014.31.1.4

Bello, M. (2001). Desplazamiento forzado y reconstrucción de identidades [Forced displacement and identity reconstruction]. Ministerio de Educación Nacional. Bogotá, DC: Instituto Colombiano para el Fomento y Desarrollo de la Educación Superior ICFES.

Beristain, C., Dona, G., Páez, D., Pérez-Sales, P. \& Fernández, I. (1999). Reconstruir el tejido social. Un enfoque crítico de la ayuda humanitaria [Rebuild the social fabric. A critical approach to humanitarian aid]. Barcelona: Icaria Editorial / Antrazy.

Bilbao, M., Páez, S., Da Costa, D. \& Martínez-Zelaya, G. (2013). Cambio en creencias básicas y crecimiento post estrés: Un estudio transversal sobre el fuerte impacto de los hechos positivos sobre el bienestar [Changes in basic beliefs and post-stress growth: A cross-sectional study on the strong impact of positive events on wellbeing]. Terapia Psicológica, 31(1), 127-139.

Blanco, A. \& Amarís, M. (2014). La ruta psicosocial del desplazamiento: Una perspectiva de género [Psychosocial pathway of displacement: A gender perspective]. Universitas Psychologica, 13(2), 661-679. http://dx.doi.org/10.11 144/Javeriana.UPSY13-2.

Blanco, A. \& Valera, S. (2007). Los fundamentos de la intervención psicosocial [The basics of psychosocial intervention]. En A. Blanco \& J. 
Rodríguez (Eds.), Intervención psicosocial. (Cap. 1). Madrid: Mac Graw Hill.

Bourdieu, P. (1986). The forms of capital. En J. Richardson (Ed.), Handbook of theory and research for the sociology of education (pp. 241-258). New York: Greenwood Press.

Cardozo, A., Amarís, M. \& Blanco, A. (2015). Modelo analítico de las relaciones funcionales entre el capital social, la salud mental y las cogniciones postraumáticas en víctimas del desplazamiento forzado asociado al conflicto armado [Analytical model of functional relations between social capital, mental health and posttraumatic cognitions in victims of forced displacement associated with armed conflict]. Tesis Doctoral no publicada. Universidad del Norte. Barranquilla, Colombia.

CEPAL (2014). Panorama social de América Latina [Latin American social perspective]. Organización de Naciones Unidas, Chile. Recuperado el 14 de enero de 2014 de http://repositorio. cepal.org/bitstream/handle/11362/37626/S142 0729 es.pdf.

Coleman (1994). Foundations of social theory. Belknap Press: Cambridge, M.A.

Cortés, O.F. (2015). Well-being labor teaching in the educational public sector of Barranquilla Colombia. Procedia - Social and Behavioral Sciences, 191, 2835-2843. http://dx.doi.org/10. 1016/j.sbspro.2015.04.660

Departamento Administrativo Nacional de Estadística [DANE]. (2014). Boletín técnico: Pobreza monetaria [Technical bulletin: Monetary poverty]. DANE: Bogotá, Colombia. Recuperado el 30 de marzo de 2015 de http://www.dane. gov.co/files/investigaciones/condiciones_vida/p obreza/bol_pobreza_mon_jul13_jun14.pdf.

Díaz-Albertini, J. (2001). Capital social, organizaciones de base y el estado. Recuperando los eslabones perdidos de la sociabilidad [Social capital, grassroots organizations and the state. Recovering the missing links of sociability]. Trabajo presentado en la Conferencia Regional sobre Capital Social y la Reducción de la Pobreza en América Latina y el Caribe. Cepal: Santiago de Chile. Recuperado el 16 de marzo de 2015 de http://www.cepal.org/prensa/ noticias/ comunicados/3/7903/diazalbertini.pdf.

Díaz, D., Blanco, A., Horcajo, F. \& Valle, C. (2007). La aplicación del modelo del estado completo de salud al estudio de la depresión [The application of the model of the complete health status to the study of depression]. Psicothema, 19(2), 286- 294.

Díaz, D., Rodríguez-Carvajal, R., Blanco, A., Moreno-Jiménez, B., Gallardo, I., Valle, C. \& Van Dierendonk, D. (2006). Adaptación española de las Escalas de Bienestar Psicológico de Ryff [Spanish adaptation of Ryff's Psychological Well-Being Scales]. Psicothema, 18(3), 572577.

Diener, E. (1984). Subjective well-being. Psychological Bulletin, 95(3), 542-575.

Diener, E. (1994). Assessing subjective well-being. Progress and opportunities. Social Indicators Research, 31, 103-157.

Diener, E. (2005). Guidelines for national indicators of subjective well-being and ill-being. Journal of Happiness Studies, 7(4), 397-404. http://dx.doi.org/10.1007/s10902-006- 9000-y.

Durkheim, E. (2012). El suicidio [The suicide]. Madrid: Ediciones AKAL S.A.

Durston, J. (2000). ¿Qué es el capital social comunitario? [What is community social capital?]. División de Desarrollo Social. Santiago de Chile: Naciones Unidas. CEPAL - Serie Políticas Sociales, 38. Recuperado el 20 de noviembre de 2013 de http://repositorio.cep al.org/bitstream/handle/11362/5969/1/S0 007 574_es.pdf.

Durston, J. (2002). El capital social campesino en la gestión del desarrollo rural. Diadas, equipos, puentes y escaleras [The peasant social capital in the management of rural development. Dyads, equipment, bridges and stairs]. Santiago de Chile: CEPAL. Recuperado el 24 de octubre 
de 2014 de http://archivo.cepal.org/pdfs/2002/ S2002033.pdf.

Fernández, J., Manrique, B.S., Bojorquez, I. \& Salinas, A. (2014). Inc ome inequality, socioeconomic deprivation and depressive symptoms among older adults in Mexico. PLoS ONE, 9(9), 24. http://dx.doi.org/10.1371/jour nal.pone.0108127

Forni, P., Siles, M. \& Barreiro, L. (2004). ¿Qué es el capital social y cómo analizarlo en contextos de exclusión social y pobreza? Estudios de caso en Buenos Aires, Argentina [What is social capital and how to analyze it in contexts of social exclusion and poverty? Case studies in Buenos Aires, Argentina]. JSRI Research Report, 35, 1-20. Recuperado el 18 de junio de 2015 de http://www.jsri.msu.edu/upload/resear ch- reports/rr35.pdf.

González, R. (2009). Capital social: Una visión introductoria a sus principales conceptos [Social capital: An introductory vision to its main concepts]. Revista Latinoamericana de Ciencias Sociales, Niñez y Juventud, 7(2), 17311747.

Hanifan, L.J. (1916). The rural school community center. Annals of the American Academy of Political and Social Science, 67, 130-138. http://dx. doi.org 10.1177/000271621606700118.

Hurtado, D., Kawachi, I. \& Sudarsky, J. (2011). Capital social y autopercepción de la salud en Colombia: lo bueno, lo malo y lo feo [Social capital and self-perception of health in Colombia: The good, the bad and the ugly]. Social Science and Medicine, 72(4), 584-590. Recuperado el 23 de septiembre de 2014 de http:// www.socialcapitalgateway.org/content/paper/ hurtado-d- kawachi-i-sudarsky-j-2011-socialcapital-and-self-rated-health-colombia-go.

Hurtado, D., García, M. \& Copete, A. (2013). Las vicisitudes del capital social de Colombia. Los cambios de ksocial (capital social), Confie (Confianza Institucional y control Indirecto del Estado) y Fenoval (Fe en Fuentes de Informa- ción No Validadas), 1997, 2005, 2011 [Vicissitudes of the social capital of Colombia. The changes of ksocial (social capital), Confie (Institutional Confidence and Indirect State control) and Fenoval (Faith in Unrecorded Information Sources), 1997, 2005, 2011]. Bogotá: Fundación Antonio Restrepo Barco.

Kawachi, I., Subramanian, S. \& Kim, D. (2008). Social capital and health. Springer: New York.

Keyes, C. (1998). Social well-being. Social Psychology Quarterly, 61(2), 121-140.

Keyes, C. (2005a). Health as a complete state: The added value in work performance and healthcare costs. Journal of Environmental Medicine. 47(5), 523-532.

Keyes, C. (2005b). Mental illness and/or mental health? Investigating axioms of the complete state model of health. Journal of Consulting and Clinical Psychology, 7(3), 539-548.

Keyes, C., Shmotkin, D. \& Ryff, C. (2002). Optimizar el bienestar: El encuentro de dos tradiciones empíricas [Optimizing well-being: The meeting of two empirical traditions]. Diario de Personalidad y Psicología Social, 82(6), 007 1022.

Kripper, C. \& Sapag, J. (2009). Capital social y salud en América Latina y el Caribe: Una revisión sistemática [Social capital and health in Latin America and the Caribbean: A systematic review]. Revista Panamericana de Salud Pública, 25(2), 162-170.

Landini, F.P. (2012). Expectativas de los agentes de desarrollo rural argentinos sobre la psicología y sobre la inserción profesional de los psicólogos en el ámbito de la extensión rural [Argentine rural development agents' expectations about psychology and about the employability of psychologists in the area of rural extension]. Interdisciplinaria, 29(2), 359-377. Recuperado el 10 de mayo de 2016 de http://www.redalyc. org/articulo.oa? id=18026361010.

León, O. \& Montero, I. (2004). Métodos de investigación en psicología y educación [Research 
methods in Psychology and education]. Madrid: Mc Graw Hill.

López, A. \& Sánchez, E. (2001). Estructura social, apoyo social y salud mental [Social structure, social support and mental health]. Psicothema, 13(1), 17-23.

Madariaga, C. \& Sierra, O. (2000). Redes sociales y pobreza [Social networks and poverty]. Psicología desde el Caribe, 5(1), 127-156.

Martín-Baró, I, (1988). La violencia política y la guerra como causas del trauma psicosocial en El Salvador [Political violence and war as causes of psychosocial trauma in El Salvador]. Revista de Psicología de El Salvador, 9(1), 123-141.

Monsegur, S., Espinosa, A. \& Beramendi, M. (2014). Identidad nacional y su relación con la dominancia social y la tolerancia a la transgresión en residentes de Buenos Aires (Argentina) [National identity and its relationship with social dominance and tolerance transgression in Buenos Aires' residents (Argentina)]. Interdisciplinaria, 31(1), 5-23. Recuperado el 8 de mayo de 2016 de http://www.scielo.org.ar/pdf/interd/ v31n1/v31n1a01.pdf;; https://doi.org/10.1688 8/interd.2014.31.1.1

Muñoz, Y., Poblete, Y. \& Jiménez, A. (2012). Calidad de vida familiar y bienestar subjetivo en jóvenes con discapacidad intelectual de un establecimiento con educación especial y laboral de la ciudad de Talca [Quality of family life and subjective well-being in young people with intellectual disability in school with special education of the Talca city]. Interdisciplinaria, 29(2), 207-221. Recuperado el 9 de mayo de 2016 de http://www.redalyc.org/pdf/180/180 26361003.pdf.; https://doi.org/10.16888/ interd. 2012.29.2.1

Organización Mundial de la Salud [OMS]. (1948). Official records of the world health organization. Geneva: World Health Organization. Recuperado el 23 de abril de 2016 de http:// www. who.int/library/collections/historical/es/ind ex3. html
Organización Mundial de la Salud [OMS]. (1978). Carta de Alma Ata sobre atención primaria en salud [Alma Ata letter on primary health care]. Ginebra, Suiza: OMS.

Organización Mundial de la Salud [OMS]. (1986). Carta de Ottawa sobre promoción de la salud [Ottawa letter on Health Promotion]. Ottawa, Canadá: OMS.

Organización Mundial de la Salud [OMS]. (1997). Carta de Yakarta sobre promoción de la salud en el siglo XXI [Jakarta Charter on Health Promotion in the 21st Century]. Yakarta, Indonesia: OMS.

Organización Mundial de la Salud [OMS]. (2004). Promoción de la salud mental. Conceptos, evidencia emergente, práctica: Informe compendiado [Promotion of mental health. Concepts, emerging evidence, practice: Summary report]. Organización Mundial de la Salud, Departamento de Salud Mental y Abuso de Sustancias en colaboración con la Fundación Victoriana para la Promoción de la Salud y la Universidad de Melbourne, Australia: OMS.

Organización Mundial de la Salud [OMS]. (2005). Carta de Bangkok sobre promoción de salud en un mundo globalizado [Bangkok charter on health promotion in a globalized world]. Bangkok: OMS.

Organización Mundial de la Salud [OMS]. (2013). Carta de Helsinki sobre salud en todas las politicas [Helsinki charter on health in all policies]. Helsinki, Finlandia: OMS.

Organización Mundial de la Salud [OMS]. (2015). Día mundial de la salud mental: Dignidad y salud mental [World mental health day: Dignity and mental health]. New York: OMS. Recuperado el 12 de agosto de 2016 de http://www.wh o.int/mental_health/world-mental-health-day/ 2015 infosheet/es/

Palacio, J., Abello, R., Madariaga, C. \& Sabatier, C. (1999). Estrés post-traumático y resistencia psicológica en jóvenes desplazados [Post-traumatic stress and psychological resistance in 
young displaced persons]. Investigación y Desarrollo, 10(1), 16-29.

Palacio, J., Correa, A., Díaz, M. \& Jiménez, S. (2003). La búsqueda de la identidad social. Un punto de partida para comprender las dinámicas del desplazamiento - restablecimiento forzado en Colombia [The search for social identity. A starting point to understand the dynamics of displacement - forced resettlement in Colombia]. Investigación y Desarrollo, 11(1), 26- 55.

Paredes, L. Paredes-Díaz, D., Blanco, A., Sutil, L. \& Schweiger, I. (2007). Argumentos para una propuesta psicosocial del trauma II: El bienestar, patrón de salud y de trastorno [Arguments for a psychosocial proposal of trauma II: Well-being, pattern of health and disorder]. Convivir con la Violencia, 1, 17-45.

Putnam, R.D. (1993). The prosperous community: Social capital and public life. The American Prospect, 13, 35-42.

Putnam, R. (1995). Bowling alone: The diminution de la America's capital social. Journal of Democracy, 6(1), 65-78.

Ramos-Vidal, I., Holgado, D. \& Maya-Jariego, I. (2014). Las redes personales de los desplazados internos por la violencia política en Colombia: Una aproximación al caso del Departamento del Atlántico [The personal networks of the internally displaced by political violence in Colombia: An approach to the case of the Atlantic Departament]. TRACE, 65, 51-68.

Resett, S., Costa, D., Murata, C. \& Falcone, N. (2015). Equivalencia factorial del Cuestionario de Agresores / Víctimas de Olweus según Género [Factorial invariance of the Olweus Bully / Bullied Questionnaire according to gender]. Interdisciplinaria, 31(1), 169-181. Recuperado el 12 de mayo de 2016 de http://www. scielo.o rg.ar/pdf/interd/v32n1/v32n1a09.pdf. https://doi.org/10.16888/interd.2015.32.1.9

Rose, R. (2000). How much does social capital add to individual health? A survey study of Russians. Social Science \& Medicine, 51(9), 1421-
1435. http://dx.doi.org/10.1016/S0277-9536 (0 0)00106-4.

Ryff, C. (1989). Happiness is everything, or is it? Explorations on the meaning of psychological well-being. Journal of Personality and Social Psychology, 57(1), 1069-1081.

Sapag, J. \& Kawachi, I. (2007). Capital social y promoción de la salud en América Latina [Social capital and health promotion in Latin America]. Revista Saúde Pública; 41(1), 139-149.

Sen, A. (2011). Desarrollo y libertad [Development and freedom]. Barcelona: Editorial Planeta.

Sudarsky, J. (1999). El capital social en Colombia. La medición nacional con el Barcas [The social capital in Colombia. The national measurement with the Barcas]. Separata No. 5 de 5. República de Colombia, Departamento Nacional de Planeación. Unidad de Análisis Macroeconómico. Archivos de Macroeconomía. Recuperado el 25 de mayo de 2014 de https://colabora cion.dnp.gov.co/CDT/Estudios\%20Econmicos/ 126.pdf.

Sudarsky, J. (2007). La evolución del capital social en Colombia, 1997-2005. Los cambios en Capital Social (Ksocial), Confianza y Control Indirecto del Estado (Confie) y Fe en fuentes de información no validadas (Fenoval) [The evolution of social capital in Colombia, 1997-2005. Changes in Social Capital (Ksocial), Confidence and Indirect State Control (Confie) and Faith in unvalidated sources of information ( $\mathrm{Fe}-$ noval)]. Bogotá: Fundación Restrepo Barco. Recuperado el 30 de mayo de 2014 de http:// www.funrestrepobarco.org.co

Sudarsky, J. (2008). Ksocial, Confíe y Fenoval en cuatro ciudades colombianas, 1997-2005: Las implicaciones para la tercera ola de la democracia y la construcción de la sociedad cívica moderna [Ksocial, Confie and Fenoval in four Colombian cities, 1997- 2005: The implications for the third wave of democracy and the construction of modern civic society]. Congreso Mundial de Ciudades, Puerto Alegre, Brasil. 
Urteaga, E. (2013). La teoría del capital social de Robert Putnam: Originalidad y carencias [Robert Putnam's theory of social capital: Originality and gaps]. Reflexión Política, 15, 44-60. Recuperado el 13 de marzo de 2014 de http://oai.redalyc.org/articulo.oa? id=11028415005.

Vargas, F.G. (2002). Hacia una teoría del capital social [Towards a theory of social capital]. Revista de Economía Institucional, 4(006), 71108.

Verduin, F., Smid, G., Wind, R. \& Scholte, W. (2014). In search of links between social capital, mental health and sociotherapy: A longitudinal study in Rwanda. Social Science \& Medicine. 121, 1-9. http://dx.doi.org/10.1016/j.socscimed. 2014.09.054.
Wind, T. \& Komproe, I. (2012). The mechanisms that associate community social capital with post-disaster mental health: A multilevel model. Social Science \& Medicine, 75(9), 1715-1720. http://dx.doi.org/10.1016/j.socscimed.2012.06.0 32.

Woolcock, M. \& Narayan, D. (2000). Social capital: Implications for development theory, research, and policy. The World Bank Research Observer, 15(2), 225- 249.

Wu, Q., Lu, D. \& Kang, M. (2014). Social capital and the mental health of children in rural China with different experiences of parental migration. Social Science and Medicine, 132 (May), 270-277. http://dx.doi.org/10.1016/j.socscim ed.2014.10.050

Facultad de Psicología Universidad de la Costa Universidad del Norte Unidad de Atención y Reparación Integral a Victimas Barranquilla - Colombia

Fecha de recepción: 27 de noviembre de 2015 Fecha de aceptación: 29 de diciembre de 2016 